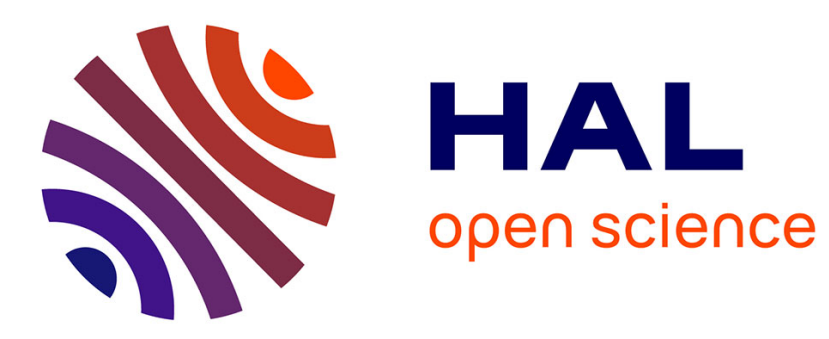

\title{
Amélioration des taillis-sous-futaie
}

Léon Schaeffer, André Schaeffer

\section{To cite this version:}

Léon Schaeffer, André Schaeffer. Amélioration des taillis-sous-futaie. Revue forestière française, 1951, 9, pp.537-551. 10.4267/2042/27804 . hal-03380118

\section{HAL Id: hal-03380118 https://hal.science/hal-03380118}

Submitted on 15 Oct 2021

HAL is a multi-disciplinary open access archive for the deposit and dissemination of scientific research documents, whether they are published or not. The documents may come from teaching and research institutions in France or abroad, or from public or private research centers.
L'archive ouverte pluridisciplinaire HAL, est destinée au dépôt et à la diffusion de documents scientifiques de niveau recherche, publiés ou non, émanant des établissements d'enseignement et de recherche français ou étrangers, des laboratoires publics ou privés. 


\section{REVUE FORESTIERE FRANÇAISE}

D'ans ce numéro:

L. et A. Schaeffer: Amélioration des taillis-sous-futaie. - F. DE Lemps: Volume critique, plan de balivage et composition normale dans les taillis-sousfutaie. - L. S.: Des classes d'âge aux classes de grosseur. - F. DE LEMPS: Futaie jardinée par bouquets et futaie de chêne par bouquets. - GuICHARD: Le chêne dans l'évolution des taillis-sous-futaie de la Haute-Saône. - G. B.: La boussole forestière.

\section{AMÉLIORATION DES TAILLIS SOUS FUTAIE}

Indice bibliographique: $\mathrm{F}$ 22.21 : 22.6I

Le traitement en taillis sous futaie passe incontestablement par une crise. Certes, il a encore ses partisans, mais il semble bien que ses adversaires se font chaque jour plus nombreux. Les reproches qui lui ont été adressés sont d'ordre économique et d'ordre cultural. Du point de vue économique, on fait ressortir que la population rurale a fortement diminué, que le bois a cessé d'être un combustible industriel, que la demande en bois d'œuvre s'accroît constamment. Du côté cultural, on attire l'attention sur l'action épuisante du régime du taillis, les remontées du plan d'eau qui succèdent aux exploitations brutales, la disparition des essences précieuses, le défaut de régénération. Loin d'être le mode de traitement de tout repos que beaucoup s'imaginent, le taillis sous futaie doit être amélioré et transformé.

Qu'est-ce qui a été fait jusqu'ici dans ce but ? Qu'est-ce qu'on peut faire dans l'immédiat ? Quelles sont les tâches de l'avenir ?

\section{I. - LES DIFFÉRENTES MÉTHODES D'AMÉLIORATION DES TAILLIS SOUS FUTAIE}

Pour améliorer les taillis sous futaie, on a imaginé plusieurs procédés : la multiplication des réserves, l'allongement des révolutions, le recours à la régénération par voie sexuée.

La multiplication des réserves répond au conseil lancé par Puton à la fin du siècle dernier: ( Faites des arbres ). Il recommandait d'augmenter graduellement l'intensité des balivages “ jusqu'au moment où le plein sera obtenu, c'est-à-dire où toute la surface sera occupée par des arbres de différentes classes »).

Les nombreux baliveaux ainsi réservés deviennent le support de l'accroissement: on diminue la place consacrée au taillis, on fait davantage de bois d'œuvre, de bois d'industrie, de chauffage de choix. L'augmentation de la valeur de la production résulte à la fois de ce 
qu'on a plus d'arbres et de ce qu'étant plus serrés, ils sont mieux élagués, ont une plus grande hauteur de fût.

Maịs, du côté cultural, il en résulte incontestablement une déformation du régime. Ce n'est souvent même plus de la futaie sur taillis: le taillis est réduit à l'état de sous-étage, de souille ou même disparait.

Après un patient travail de dépouillement d'archives rassemblées par de la Hamelinaye, H. Perrin conclut à l'existence d'un volume critique d'ailleurs très faible. Dès que la réserve comporte un volume bois d'œuvre supérieur à $20-30 \mathrm{~m}^{3}$, la disparition des chênes se manifesterait. Pour qu'il n'y ait pas'difficultés à marquer des baliveaux de chênes, les arbres ne devraient pas occuper après la coupe plus que le tiers de la surface.

Cette multiplication des réserves aboutit cependant à un accroissement du nombre des porte-graines et, à ce titre, peut aider à la production de semis. Mais, comme on le verra, tous les semis qui naissent sont loin d'être sauvés: ils ne sont même pas nécessairement tous d'une essence précieuse.

L'allongement des révolutions peut être très avantageux en ce qu'il substitue à la production de charbonnette celle de petit rondin et de bois de mine. Pratiqué dans des forêts à base de charme et de hêtre, il favorise l'installation du semis de hêtre sous le couvert relevé du taillis âgé. On pourrait citer de nombreux cas de retours massifs du hêtre s'opérant clans des taillis de charme presque pur du seul fait que la révolution a été portée à un chiffre supérieur à 30 ans.

Mais le traitement en taillis sous futaie à longue révolution ne peut être considéré comme une amélioration du régime à appliquer dans tous les cas. C'est au contraire incontestablement le passage des très courtes révolutions d'autrefois aux révolutions de 25 et 30 ans qui est à l'origine de la disparition du chêne. Les raisons en sont évidentes. D'une part, plus les révolutions sont longues, moins i1 . a de chances que le passage de la coupe coïncide avec une glan. dée. D'autre part, les semis de chêne ont à lutter plus longtemps contre la concurrence du taillis.

En outre, il ne faut pas se dissimuler que l'allongement pur et simple des révolutions ne donne pas au traitement un caractère de culture intensive: au contraire, la récolte des arbres ne se fait plus au moment le plus opportun et on est obligé de recourir à des artifices, tels que celui des bandes alternes, pour concilier par exemple l'exploitation du taillis à 50 ans avec la récolte d'arbres tous les 25 ans.

Un autre inconvénient d'un allongement des révolutions résulte de ce que, en vieillissant, le taillis s'élance, englobe la cime des réserves et fait mourir les branches bassès. Nous en reparlerons. 
Enfin l'amélioration du taillis sous futaie peut résulter d'un changement plus ou moins radical apporté au mode de reproduction. A un rajeunissement par rejets, on peut substituer la régénération sexuée, grâce aux semis naturels ou même par voie artificielle, au moyen de semis faits de main d'homme, ou de plantations. C'est ce qu'on fera notamment dans les cas suivants:

Tout en ayant des raisons de rester fidèle au taillis sous futaie, on peut être amené, quand le recrutement des baliveaux est déficitaire, à provoquer une régénération par voie de semis. On sait que dans le cas du chêne, il est nécessaire, pour arriver à un résultat, de procéder à l'extraction de futaies sur les taches de semis, au plus dans le délai de 5 ans. Ces coupes ont reçu du Comité consultatif des aménagements le nom de " coupe de rénovation ) et les forêts où doit se pratiquer ce genre d'opérations ont été étiquetées " forêts à rénover ).

La régénération par voie sexuée est aussi celle qui est recherchée dans le cas où on renonce au taillis sous futaie et adopte la solution radicale de la conversion en futaie.

La conversion en futaie peut être prévue avec substitution d'essence et l'enrésinement n'en est qu'un cas particulier. Des méthodes différentes sont à employer suivant le tempérament de l'essence à introduirc. La plus connue est la méthode des coupes d'abri d'un usage constant pour l'introduction des sapins. Avec les esscnces de grande lumière, il n'est plus question au contraire de 'loser le couvert. On opère par coupes à blanc et les dégagements roivent être conduits énergiquement et sans interruption perılant plusieurs années. De bonne heure, on sait si on a réussi ou... échoué.

Plus souvent, on pratiquera la conversion en futaie avec les essences spontanées de la forêt. Au début, on ne pensait qu'à obtenir une futaie pleine. Depuis un demi-siècle, on envisage d'autres formes de peuplements.

Quel que soit le but à atteindre, la conversion se décompose en trois opérations; oì nous retrouvons précisément les trois remèdes envisagés pour améliorer les taillis sous futaie.

Il s'agit tout d'abord d'étoffer la réserve en vue de multiplier les forte-graines. Il faut ensuite résorber le taillis, ce qui suppose son vieillissement préalable. Enfin, on fait naître les semis en vue de substituer à la régénération par rejets la régénération par voie de scmences.

Recherchoris comment il est dans la pratique fait appel à ces trois opérations.

Dans le procédé classique de conversion graduelle, on fait successivement trois sortes de coupes dont chacune répond à l'un des trois buts qui viennent d'être définis: coupes temporaires de taillis 
sous futaie, coupes préparatoires à la conversion, coupes de régénération.

La période de vieillissement du taillis conduit celui-ci en principe jusqu'à l'époque où il deviendra fertile et où les souches auront par contre perdu leur faculté de rejeter. De cette façon, on ne risque pas de voir les rejets étouffer les semis qui se développent à la suite d'un relèvement progressif du couvert ou d'une franche mise en ensemencement, et qui permettront la conversion proprement dite. Ce principe admis, les coupes préparatoires peuvent être conduites très différemment.

Sous l'influence du traitement ancien en taillis sous futaie, les fûts des réserves ont été moulés jusqu'à une certaine hauteur par le taillis; en fin de révolution, ils sont comme dans un puits.

Si le taillis n'est plus exploité à l'âge précédcmment admis, suivant l'image de $\mathrm{H}$. SAUR, la margelle du puits monte et élague tardivement les réserves. Le bois produit est de qualité médiocre: les pourritures et les trous de pics sont fréquents.

Les auteurs ont bien vu le danger, et les traités de sylviculture recommandent de dégager dans les coupes préparatoires la cime des gros chênes, mais, comme d'autre part, ils prescrivent de laisser le massif complet, les opérateurs par prudence n'osent pas toujours procéder avec l'énergie nécessaire et le mal se produit. C'est ce que certains ont pu appeler la " rançon de la conversion ", mais pour d'autres, ce n'est qu' " un point noir dans les conversions classiques $)$.

Dans le système SAur, on ne fait pas de coupes temporaires de taillis sous futaie. On ne s'en remet pas non plus aux coupes de régénération du soin de nous procurer dans un avenir lointain des peuplements susceptibles de produire des bois appréciés. Bien souvent, les éléments sont d'ores et déjà présents: il suffit de bien en tirer parti. Les seules coupes qu'on pratiquera d'emblée sur toute l'étendue de la forêt seront donc des coupes préparatoires. Elles seront l'objet de soins particuliers. Le taillis sera traité avec tous les égards dus à un sous-étage indispensable à la protection des fûts des réserves.

Quand on a une surabondance. d'essences précieuses parmi les arbres réservés, on peut extraire les plus vieux d'entre eux dès le début des coupes préparatoires et ainsi conduire jusqu'au stade des coupes de régénération des semenciers qui ne soient pas d'un âge excessif. Du même coup, les sacrifices d'exploitabilité sont réduits. C'est le système pratiqué notamment à Montargis.

On a pratiqué également sous le nom de "système AUBERT » un balivage intensif sans vieillissement du taillis. Si le taillis est riche en essences précieuses, on a directement un perchis, puis une futaie sur jeune souche, d'un aspect très satisfaisant pour peu 
qu'on ait pris la précaution de griffer un nombre suffisant de brins d'une essence de remplissage appelée à jouer le rôle d'un sousétage.

Le vieillissement du taillis n'est donc pas toujours indispensable. On l'a souvent évité pour le hêtre.

De même avec le chêne pédonculé, dont les semis démarrent souvent très rapidement, on peut l'année d'une glandée faire la coupe de régénération en recépant un taillis relativement jeune. Mais c'est au prix de dégagements répétés.

Le vieillissement des taillis est au contraire absolument indispensable quand on veut favoriser le retour du hêtre ou du sapin, bien que le nombre de semenciers de ces essences soit minime.

On parvient donc en variant les procédés, en les adaptant à chaque cas particulier, à gagner un peu de temps sur le procédé classique de conversion et à obtenir de bons résultats culturaux.

Dans bien des cas cependant, on renonce à la futaie régulière pour des raisons culturales et économiques.

Dans les parties basses et humides, on ne veut pas risquer les remontées brusques du plan d'eau qui sont la conséquence de coupes de régénération menées sur des hectares ou même des dizaines d'hectares. Dans les parties élevées, c'est l'envahissement des fougères et de la callune qui peut être à redouter. Dans les deux cas, une diminution de la productivité est possible.

Par ailleurs, les sacrifices d'exploitabilité entrainés par la mise en régénération simultanée de toute une parcelle peuvent être considéral)!es. Si la forêt présente des stations de fertilité diverses, des âges d'exploitation variés s'imposent et la régularité obtenue à grands frais pour une révolution devrait quand même être abandonnée à la révolution suivante. Dans ces conditions, autant vaut s'accommoder des inégalités d'âge entre les réserves du taillis sous futaie, et régénérer les différentes surfaces qu'elles occupent ̀̀ mesure qu'elles atteignent leur maturité. C'est donc vers une futaie irrégulière qu'on va s'orienter.

Huffel, reprenant d'ailleurs un vieux terme employé entre autres par GURNAUD, a baptisé ( futaie claire ) un peuplement formé de trois éléments: tout d'abord un peuplement principal présentant quelque analogie avec un taillis sous futaie riche en réserve, ou encore avec une futaie entr'ouverte qu'on maintiendrait en perpétuel état d'ensemencement, ensuite des semis, enfin une souille comprenant les rejets qui se forment lors des recépages fréquents portant sur tout l'étage dominé à l'exclusion des semis. Pour obtenir cette forme de peuplement, on devait passer en coupe à une rotation de Io à I 5 ans pour $y^{\prime}$ pratiquer d'un seul coup sur toute l'étendue de la parcelle les différentes opérations devant assurer la régénération et l'amélioration. 
Dans le département de la Haute-Saône, où depuis I9I3 le mouvement de conversion a pris une grande ampleur, l'expression de futaie jardinée a été employée concurremment à celle de futaie claire.

C'est l'expression de futaie jardinée qui a été préférée par $\mathrm{P}$. Marc pour le hêtre. En principe, les différences de tempérament des chênes et du hêtre justifiaient les différences de traitement qui opposent la futaie claire et la futaie jardinée. Dans la futaie jardinée feuillue, le couvert est supposé continu, l'ensemencement est obtenu sans qu'il soit nécessaire de faire des trouées, le taillis disparaît complètement sans laisser de traces.

Finalement, la terminologie adoptée pendant le deuxième quart de notre siècle a été " futaie claire ) dans le cas du chêne, " futaie jardinée » dans le cas du hêtre. Actuellement, ces deux dénominations tendent à céder la place à celle de "futaie par bouquets ).

Comme on le voit, les méthodes d'amélioration entre lesquelles nous avons à choisir sont nombreuses. Les plus récentes d'entre elles sont la coupe de rénovation et la futaie par bouquets. Elles méritent qu'on cherche à préciser leurs règles culturales et à fixer les procédés d'aménagement qui leur sont applicables.

\section{II. - RÈgles CUlturales}

\section{A. - Coupes de rénovation.}

Quelles sont les forêts à rénover et à quelle technique doit-on avoir recours?

Puisque la difficulté de recrutement des baliveaux est la manifestation d'un défaut de régénération clu chêne, il faut "rénover ) toutes les forêts où se constate un ralentissement de cette régénération.

Quant à la technique, elle consiste essentiellement dans les coupes récemment exploitées à extraire quelques vieux anciens, sur " glandée acquise ", c'est-à-dire dès que la qualité des semences a $\mathrm{pu}$ être constatée. Une variante consiste en outre à découvrir les taches de semis dans les vieilles coupes dont l'exploitation est prochaine.

Une instruction administrative précise que les coupes de rénovation peuvent porter sur un groupe de 5 coupes exploitées ou à exploiter à brève échéance.

Strictement donc, seules les forêts où les chênes forment la réserve peuvent être soumises à des coupes de rénovation. Puisqu'il s'agit de sauver des semis de chêne, il faut faire des trouées assez grandes, agrandir les petites trouées déjà existantes et en faire des clairières de 30 à 80 mètres de diamètre. Pour arriver au résultat avec le moins de sacrifices d'exploitabilité possible, il peut être indiqué d'enlever les arbres séparant deux ou trois trouées voisines. La 
clairière créée de cette façon est assez vaste pour que la lumière arrive à flots et qu'on n'ait plus besoin ensuite de l'étendre: tous les soins à donner se limiteront alors aux dégagements de semis.

L'opération a surtout été prévue pour le cas des chênes, car c'est pour cette essence que le défaut de régénération est le plus manifeste. Mais on ne voit pas pourquoi la solution ne serait pas étendue au hêtre. Il ne serait pas alors question bien entendu d'une opération aussi radicale et la coupe de rénovation y prendrait seulement le caractère d'une coupe d'ensemencement ou d'une coupe secondaire. Trop souvent, on a dû se résigner à déplorer la disparition de semis de hêtre, en faveur desquels rien n'avait été fait.

Supposons l'opération pratiquée dans de bonnes conditions. Pour qu'elle soit véritablement couronnée de succès, il faut que les taches de régénération ainsi obtenues soient suivies. Il faut donc qu'elles soient repérées sur un croquis à grande échelle de la parcelle. On peut même les délimiter sur le terrain en ceinturant les arbres à la peinture à $2 \mathrm{~m}$. de haut.

Les dégagements de semis sont pratiqués ensuite. De Lemps, à juste titre a dit qu'ils devaient être " violents, concentrés et répétés ).

Dès que les semis ont atteint le stade du gaulis, il faut leur permettre de s'étoffer et le dégagement tournera progressivement à l'éclaircie.

Malgré les précautions, il faut bien reconnaitre que, quand la coupe reviendra en tour à la fin de la révolution, on n'y trouvera pas encore de vrais baliveaux. L'opération ne sera encore que " prometteuse de baliveaux ". La tache de gaulis passant par places au bas-perchis devra recevoir encore beaucoup de soins. L'opération levra être continuée pendant une seconde révolution.

Comme l'a dit DE Lemps, " dégagement de semis et coupes de rénovation doivent bien entrer définitivement dans la technique normale du traitement en taillis sous futaie et ne pas être réduits à un correctif intermittent du déficit des baliveaux »).

C'est le moment de citer un entrefilet quelque peu prophétique, paru en I93I daris le Bulletin de la Société forestière de Franche-Comté, intitulé: "Comment le chêne revient 》 et dû à la plume de l'Inspecteur de Luxeuil. L'auteur, M. GurtTard, y explique que, grâce à une " coupe d'amélioration " et à des dégagements, il est arrivé, dans la belle forêt communale de Saulx, à sauver des semis de chêne sous un taillis de charme de 25 ans dominé par une réserve, anciens et modernes, de 80 sujets à l'hectare, dont seulement 50 chênes. Cette opération réussie permettait à l'auteur de conclure: "Que nous voilà loin de la méthode du taillis sous futaie avec ses 25 ou 30 coupes soumises à une seule intervention tous les 25 ou 30 ans, laissant ainsi à la nature, pour une trop grande part, le soin de reconstituer les peuplements $)$. 


\section{B. - Futaie par bouquets}

Quand il s'agit de hêtres, le traitement en futaie par bouquets a beaucoup de points communs avec le jardinage.

Le mélange intime de tous les âges n'est cependant guère souhaitable. Les hêtres qui poussent plus ou moins isolés prennent des formes médiocres, peu favorables à la production de bois de qualité. La fragilité de leur écorce les expose aux coups de soleil. On aura de meilleurs résultats en les éduquant à l'état groupé, et la substitution de 1'appellation " futaie par bouquets » à celle de " futaie jardinée ») paraît très heureuse.

Pour le chêne, l'abandon du traitement en " futaie claire » est encore plus justifié. Dans l'esprit de son auteur, il devait présenter les avantages suivants: I) coïncidence fréquente entre le recépage de la souille et la production des glandées; 2) protection des semis installés contre la concurrence du taillis réduit à l'état de ( souille »; 3) occupation plus importante du sol par des arbres susceptibles de fournir du bois d'œuvre; 4) meilleur élagage des réserves du fait de leur rapprochement et plus grande hauteur du fût; 5) réalisation des arbres dépérissants dès qu'ils feront preuve d'une végétation ralentie. Ces avantages devaient somme toute résulter de ce que le nouveau traitement combinait deux opérations: une multiplication des réserves et un abaissement de la durée de la révolt1tion.

En fait, le recépage de la souille est onéreux, inutile et même dangereux, puisqu'on détruit ainsi périodiquement un sous-étage. En outre, il est trompeur de compter sur des coupes marquées à rotation fixe pour provoquer la régénération: ce sont les glandées qui doivent déclencher les coupes de régénération. Enfin, il est encore plus illusoire qu'avec le hêtre de chercher un mélange pied à pied d'arbres de différents âges.

Cuif avait déjà proposé de substituer à la futaie claire un traitement qu'il appelait " futaie pleine par bouquets ). On a simplifié l'expression en celle de "futaie par bouquets ", mais celle de CuiF se comprenait jusqu'à un certain point.

La rareté des bonnes années de fructification, l'intolérance des semis vis-à-vis du couvert, amènent à traiter les bouquets comme des placeaux de futaie régulière, en brusquant s'il le faut la coupe définitive et en assurant en temps utile une protection contre l'envahissement des arbres voisins.

Pour atteindre ce résultat, on ne peut pas compter sur une coupe analogue à une coupe de jardinage et assise de proche en proche, car elle risquerait d'arriver à contre-temps. On est donc amené à disjoindre dans le cas du chêne la coupe d'amélioration de la coupe de régénération, un peu comme il a fallu imaginer pour le taillis 
sous futaie, à côté de la coupe normale, une opération de rénovation orientée spécialement vers la régénération par voie de semis.

La coupe d'amélioration peut être prévue par contenance à rotation relativement longue: I5, même 18 ans. Elle ne doit qu'exceptionnellement interrompre le massif.

De leur côté, les coupes de régénération ne sont assujetties qu'à un mode d'assiette très souple. Elles ne doivent intervenir que sur un ensemencement dense bien installé. Une possibilité fixée par volume, avec faculté de différer ou d'avancer les coupes de 5 ans, leur convient parfaitement.

\section{III.- Procédés d'AMÉnagement}

Coupes de rénovation et conversion en futaie par bouquets ont incontestablement de grands avantages culturaux. Mais ces procédés nouveaux ne doivent pas devenir le prétexte à des exploitations abusives. Pour ne pas risquer d'appauvrir la forêt, il faut fixer une limite aux réalisations, soit qu'on calcule une possibilité en se basant sur un inventaire général, soit qu'on trouve une autre façon de sauvegarder l'avenir.

\section{A. - La possibilité}

De nombreux procédés ont vu le jour: ils s'inspirent tous plus nu moins des procédés déjà en usage pour la futaie régulière, parfois même de la combinaison de plusieurs procédés.

Le premier à citer est celui qui figure dans l'instruction administrative $n^{\circ} 74$ en date du 25 juillet I950 et que rappelle l'instruction $\mathrm{n}^{\text {’ }} 37$ du I7 mai I95I: “ La surface à parcourir en coupes de rénovation n'excédera pas le $\mathrm{I} / 5$ de chaque parcelle $)$. Il a été objecté à cette prescription qu'il n'était pas facile d'apprécier la surface parcourue par une coupe. Mais il ne s'agit pas d'un travail de topographie, et, pour connaitre la surface que couvraient lesi arbres extraits, :1 est bien suffisant de la déterminer au moyen d'un tarif qui donne la surface couverte en se basant par exemple sur la formule empirique:

$$
\mathrm{S}=\frac{\pi}{4}(20 \mathrm{~d}+\mathrm{I}, 50)^{2}
$$

d étant le diamètre à hauteur d'homme exprimé en mètres, S la surface couverte en mètres carrés.

Voilà donc une méthode qui serait à classer parmi les méthodes par contenance pure.

Mais, partant de la surface, on peut arriver au volume. Dans le cas d'une futaie de chêne par bouquets, la possibilité des coupes de régénération se calculera en faisant le raisonnement suivant: 
L'âge d'exploitation est fixé à I 50 ans. La coupe de régénération unique portera donc en moyenne chaque année sur I/I50 de la forêt. Pour transformer cette possibilité-contenance en possibilitévolume, on se base sur le fait d'expérience que les trouées de régénération pratiquées à bon escient sous les arbres les plus âgés entrainent par exemple sur $\mathrm{I}$ ha la réalisation de $200 \mathrm{~m}^{3}$. Dans le cas d'une forêt de I 50 ha, c'est précisément I ha et par conséquent $200 \mathrm{~m}^{3}$ qu'il faudra prendre en moyenne chaque année.

Si on s'est fixé I 5 ans comme durée d'application de l'aménagement, on peut assimiler cette durée à une période. L'ensemble des trouées de régénération portera sur I/Io de la surface. Nous voici tout près de la méthode de l'affectation unique, où période et affectation seraient respectivement le r/ro de l'âge d'exploitation et de la contenance de la série.

Reste à fixer la possibilité des coupes d'amélioration. On peut la fixer à la réalisation d'une fraction du matériel total inventorié. Comme le plus souvent, le matériel dont on part est sensiblement inférieur au matériel normal. le taux de réalisation à adopter pourra être de l'ordre de $0,5 \%$. Au total, dans la possibilité globale, la part de l'amélioration restera souvent inférieure à celle de la régénération.

$\mathrm{Si}$ on envisage l'adoption d'une possibilité par volume, on est tenté de faire appel à la méthode de 1883 et, effectivement, on peut de cette manière, quand on dispose d'un inventaire général, chiffrer une possibilité raisonnable. Dans un exemple récent, on est. arrivé à un bon recoupement de la méthode précédente en adoptant des taux indiqués par PerRin: $\mathrm{t}=\mathrm{I}, 5 \% \quad \mathrm{t}^{\prime}=3$. Quand à $\mathrm{I} / \mathrm{q}, \mathrm{la}$ forêt étant encore pauvre, on l'a pris égal à I/Io.

Mais le plus souvent, il n'est pas possible, faute de temps ou de crédits, de faire un inventaire complet de la forêt. Il faut alors se contenter de procédés expéditifs, faisant seulement intervenir les arbres qui ont atteint l'âge de majorité: les " anciens ». C'est une donnée qui se retrouve assez souvent en compulsant les archives: calepins de balivage, inventaires avant martelage, etc...

La formule de $\mathrm{I} 883$, réduite à ses deux premiers termes, peut donner la possibilité-volume en anciens. Mais on peut aussi, tout en gardant le principe de la note de 1883 , aboutir à une possibilité en nombre d'anciens, en raisonnant directement sur les nombres d'arbres.

Dans ce calcul très simple, on ne considère que le nombre des arbres majeurs. En ce qu'il ne fait aucune différence entre le simple adulte et le vieillard, le procédé est certes plus grossier que le précédent. Par contre, un perfectionnement peut être apporté au procédé de I883. Au lieu d'adopter d'une manière régide comme taux de réalisation des gros bois $\frac{\mathrm{I}}{\mathrm{H}}$, on peut adopter une fraction $\mathrm{I} / \mathrm{m}$, $\mathrm{n} / 3$ 
m étant le temps qui s'écoule entre la simple majorité de l'arbre et sa vieillesse.

Plus la station sera fertile, plus on lui demandera de produire de gros arbres et, malgré une plus grande vitesse de croissance, on donnera, à $m$ une valeur plus élevée. Elle sera par exemple de 75 ans. Cette valeur descendra à 60,50 et même 40 ans, si la station ne se prête pas à la production de vraiment gros arbres.

Il faut souhaiter qu'un jour on introduise ce perfectionnement dans le procédé de i883. Qu'une bonne fois, on adopte une coupure fixe entre les bois moyens et les gros bois et qu'on fasse seulement varier $\mathrm{m}$ !

Quant les nouveaux traitements auront été appliqués assez longtemps, grâce aux comparaisons d'inventaires, nous aurons une connaissance plus complète de la production de nos forêts. On pourra leur faire application de la méthode du contrôle. Pour le moment, il ne s'agit que de " possibilité de début ». La simple fixation d'un taux maximum de réalisation a, dans bien des cas, rendu de grands services. Ce sera par exemple, si on prévoit une coupe de jardinage et si la rotation des coupes est fixée à une douzaine d'années (cas assez fréquent pour le hêtre):

- I5\% si le volume recensé avant la coupe est inférieur à $120 \mathrm{~m}^{3}$,

- $20 \%$ si ce volume est compris entre $\mathrm{I} 20$ et $150 \mathrm{~m}^{3}$,

$-25 \%$ s'il est compris entre 150 et $200 \mathrm{~m}^{3}$,

- $30 \%$ enfin s'il est supérieur à $200 \mathrm{~m}^{3}$.

$\mathrm{Si}$ on admet que le taux de réalisation de $30 \%$ enlève sensiblement la production dans une forêt normale où on passe en coupe tous les Io ou I 2 ans, on reconnaît du fait même que le volume de $200 \mathrm{~m}^{3}$ à 1 'ha est à peu près normal. C'est donc une autre idée qui est alors abordée: celle de la fixation de la possibilité par comparaison à une composition normale.

\section{B. - La composition normale}

On connait d'une manière satisfaisante, depuis DE LIOcourT, la loi à laquelle satisfont lès compositions normales de futaies jardinées. Une bonne partie de ce qui a été établi pour les sapinières s'applique également aux hêtraies. On fait seulement observer que la densité normale du hêtre n'étant que d'environ $2 / 3$ à $3 / 4$ de celle dı sapin, on tablera sur des surfaces terrières qui, seront seulement de $\mathrm{I} 8$ à $20 \mathrm{~m}^{2}$, et des volumes totaux de 200 à $250 \mathrm{~m}^{3}$ par ha.

Le coefficient de gradation ne pourra pas descendre à I,3 ou I,4 comme dans les bonnes stations pour le sapin; il sera plutôt de 1,5 pour des diamètres de 5 en 5 et même de 1,7 pour des circonférences de 20 en 20 . Cette valeur plus forte du coefficient de gradation est la conséquence de la tendance beaucoup plus marquée chez 
le hêtre que chez le sapin de former des bouquets plus ou moins équiennes.

Pour déterminer une composition normale de futaie de chêne par bouquets, on peut, à défaut d'autre méthode basée sur l'expérience, avoir recours au procédé déjà utilisé pour la futaie claire.

Commencer par accorder aux semis, fourrés et gaulis, une certaine fraction de la surface, par exemple 20 ares sur $\mathrm{I}$ ha.

Assimiler les catégories de diamètre à des classes d'âge et répartir également les 80 ares restants entre les catégories de grosseurs. $\mathrm{Si}$, par exemple, nous cherchons à produire des arbres de $70 \mathrm{~cm}$ de diamètre, nous aurons I 2 catégories et la surface attribuée à chaque catégorie sera de $8.000 / 12=667 \mathrm{~m}^{2}$. De la surface couverte, on remonte ensuite au nombre d'arbres en divisant le couvert de la catégorie par le couvert d'un arbre.

Dans le cas d'un taillis sous futaie en excellent sol, la composition normale peut se ressentir de la longueur de la révolution. La reproduction se fait par vagues et la répartition entre les classes de grosseur n'a pas l'air d'obéir à la loi de continuité. Si la circonférence s'accroît de $60 \mathrm{~cm}$ pendant la révolution, les plus petits arbres réservés ont déjà la dimension de " modernes ». C'est ce que $\mathrm{DE}$ LEMPS a bien fait ressortir en établissant ses compositions normales correspondant aux différentes classes de fertilité.

Une fois lá composition normale précisée, la fixation de la possibilité $\mathrm{P}$ se fait par application de la formule:

$$
\mathrm{P}=\mathrm{a}+\frac{\mathrm{R}-\mathrm{N}}{\mathrm{q}}
$$

où a est l'accroissement,

$\mathrm{R}$ le matériel réel,

$\mathrm{N}$ le matériel normal,

q la durée accordée à la réalisation de l'état normal.

$\mathrm{Si} \mathrm{R}$ et $\mathrm{N}$ sont des volumes, a est la production en volume de la forêt normale.

$\mathrm{Si} \mathrm{R}$ et $\mathrm{N}$ sont des nombres d'arbres, a est le « passage à la futaie ).

Mais on peut encore se servir des compositions normales avec une idée un peu différente. Prenons le cas d'un taillis sous futaie: Une parcelle a été exploitée depuis moins de 5 ans. La question se pose de savoir s'il est opportun d'y marquer une coupe de rénovation. Mais un simple rapprochement entre la réserve effectivement marquée et la composition normale, montre qu'il n'y a pas seulement déficit de baliveaux, mais également très peu de modernes et d'anciens. Ce n'est pas le moment d'appauvrir encore la parcelle. Le remède est ailleurs: il faut planter ou semer dans les nombreux vides. 
Au contraire, vcilà une parcelle qui vient en tour normal d'ex p'oitation. Les vieilles réserves sont nombreuses. mais il n'y a pas eu de glandée. S'il en survient une d'ici 2 ou 3 ans. on pourra y faire de la rénovation. Fn attendant, il ne faut pas craindre de faire une réserve surabondante, supéricure à ce qu'indique la composition normale. Ce n'est qu’à sette condition qu'on aura beaucoup de semis et qu'on pourra revenir dans la parcelle extraire des réserves en nombre suffisant pour faire un lot vendable.

\section{IV. - De la RÉnovation a la conversion}

Tout en recommandant de donner plus d'ampleur au mouvement de conversion, il convient de mettre en garde contre une solution de facilité dont les résultats peuvent être en réalité fort différents de ceux qu'on espérait.

Il est arrivé que, pour convertir des taillis sous futaie, on ait réservé beaucoup de modernes, griffé un nombre très considérable de baliveatix, mais que, pour éviter la réclamation des communes contre ces balivages serrés, on ait sacrifié en masse les anciens. Appliqué à des peuplements situés en bon sol et où les essences précieuses abondent. le système a donné un résultat qui n'était pas mauvais: on avait plus ou moins consciemment pratiqué le système AURERT. Mais, appliqué en mauvais sol à des peuplements pauvres en essences précieuses, ce principe a conduit parfois à griffer en réserve jusqu'à $95 \%$ de charmes, cerisiers, érables champêtres, et à conserver $70 \%$ de ces essences parmi les modernes, le nombre des anciens étant extrêmement réduit.

Quinze ans après, on s'apercevait que les bons porte-graines étaient clairsemés, que les modernes charmes avaient abondamment fructifié, que les baliveaux ne s'étaient pas étoffés sur un sol maigre et n'aváient pas empêché le taillis de rejeter. Il ne pouvait donc pas être question de revenir en coupe au bout de ce laps de temps avec l'espoir de s'acheminer rapidement vers la futaie.

Les circonstances de guerre ont souvent incité à pratiquer la méthode exactement cpposée. Des exploitations de coupe par anticipation étaient imposées. Mais, s'il était bien évident qu'il fallait livrer à la consommation des villes le plus de chauffage possible, il était du devoir de ceux qui marquaient les coupes de réduire au contraire les livraisons de bois d'œuvre susceptibles de profiter à l'occupant. Le griffáge des baliveaux se fit donc léger, les modernes, charme, érable furent sacrifiés par moitié, mais les anciens furent tous gardés, à l'exception seulement de ceux qui présentaient des tares manifestes.

Il est mairitenant possible de repasser dans de telles coupes, car on a eu la chance de bénéficier en I944, 46 et 49 de glandées ou 
de faînées et les porte-graines sont nombreux. La coupe à marquer s'apparente de très près à une coupe de rénovation.

Les coupes de rénovation sont donc doublement avantageuises. D'une part, elles permettent de tirer parti des bonnes années de fructification et notamment de l'exceptionnelle glandée de I949. D'autre part, elles peuvent être substituées aux coupes trop jeunes qui ainsi attendront leur âge normal d'exploitation.

Mais elles peuvent encore avoir un rôle plus important à jouer.

Il est rare en effet qu'une forêt soit tout entière bonne ou tout à fait mauvaise. Quand elle est un peu grande, surtout si elle est morcelée, il arrive que le sol et l'exposition changent. On est amené à y faire deux parts: une qui est digne d'être traitée en conversion, une autre qui restera longtemps encore, peut-être toujours, soumise au régime du taillis. Dans les bons cantons, on a depuis plusieurs révolutions, balivé fort serré. Ailleurs, on n'a pu garder que de médiocres réserves clairsemées. Le temps est venu de prendre une décision assurant la meilleure utilisation des moyens de production: il s'agit de bâtir un aménagement qui consacre les efforts de générations de baliveurs consciencieux et tienne compte de l'évolution des mairchés.

La solution qui s'impose est la création de deux séries. Dans le cas de forêts communales, le quart en réserve est souvent tout indiqué pour constituer une de ces deux séries. Dans la série de conversion, on passera fréquemment, en moyenne tous les io ans. Dans la série de taillis sous futaie, il yı aura peut-être intérêt à allonger la révolution et à la porter à 35 ou 40 ans.

Voilà une innovation facile à faire admettre par une municipalité éclairée. Mais il est de nombreuses communes qui ne consentent pas à sortir des sentiers battus. Le maire comprend bien et acquiesce; malheureusement, deux ou trois vieux conseillers influents restent fermement hostiles. Va-t-on laisser s'étioler de belles parcelles dans lesquelles les semis abondent ? Va-t-on laisser se trouer les vieilles futaies à tronc court et à houppier important que l'état serré élague trop tard et condamne aux attaques du pic ?

Une coupe de rénovation judicieusement prescrite permettra, à défaut d'aménagement, d'exécuter l'opération qui s'impose, l'avenir de la parcelle sera sauvegardé. Le peuplement pourra attendre io ans un renouvellement du conseil municipal... à moins que, conquise par cette manne qui lui tombe du ciel, une majorité ne se dessine en faveur de la révision de l'aménagement. La coupe de rénovation aura été dans les deux sens du mot l' " amorce ) de la conversion.

Le taillis sous futaie a pu être considéré pendant plus d'un siècle comme (" le mol oreiller pour une tête bien faite ). Incontestable- 
ment, il a besoin de subir des transformations. Qu'on se contente de le rénover ou qu'allant plus loin on le soumette à une véritable conversion, nous voici à un tournant de l'histoire du traitement de nos forêts. Il nous faut innover. A notre génération de prendre ses responsabilités !

Léon et André Schaeffer.

\section{Appendice}

L'exposé qui précède a été fait aux participants de la « Semaine d'Information 》 qui s'est déroulée à Nancy du 2 au 7 avril I95I (Rev. forest. franç., mai I95I, p. 382).

Certains passages techniques de cet exposé appellent quelques explications ou renvois à des textes plus détaillés :

Composition normale des taillis-sous-futaie et rénovation. - On trouvera toutes précisions à cet égard dans l'article de F. DE LEMPS qui suit immédiatement le présent article.

Futaie de chêne par bouquets. - C'est l'aménagement de la forêt domaniale de Mirebeau (Côte-d'Or), par F. DE Lemps, qui a servi d'exemple. Le petit article qui se trouve ci-après pages $577-578$ est tiré des * règles de culture 》de cet excellent travail.

Formule de I883. - Rappelons enfin que la formule dite de 1883 donne la possibilité $\mathrm{P}$ en fonction de:

$\mathrm{n}=$ âge moyen d'exploitation des vieux bois,

$\mathrm{V}=$ volume des vieux bois,

$\mathrm{t}=$ taux d'accroissement des vieux bois,

$\mathrm{M}=$ volume des bois moyens,

$\mathrm{t}^{\prime}=$ taux d'accroissement des bois moyens.

$\mathrm{I} / \mathrm{q}=$ fraetion de cet accroissement à réaliser en coupe.

$$
\mathrm{P}=\frac{\mathrm{V}}{\mathrm{n} / 3}+\frac{\mathrm{I}}{2} \mathrm{Vt}+\frac{\mathrm{I}}{\mathrm{q}} \mathrm{M} \mathrm{t}^{\prime}
$$

